\title{
Fusing visual and inertial sensing to recover robot egomotion
}

\author{
Guillem Alenyà \\ Robosoft \\ Teknogunea Izarbel \\ 64210 Bidart, France \\ guillem_alenya@coeic.org
}

\author{
Elisa Martínez \\ CiTS La Salle. Universitat Ramon Llull \\ Pge. Bonanova 8 \\ 08022 Barcelona \\ elisa@salleurl.edu
}

\author{
Carme Torras \\ Institut de Robòtica (CSIC-UPC) \\ Llorens i Artigues 2-4 \\ 08028 Barcelona \\ ctorras@iri.upc.es
}

\begin{abstract}
A method for estimating mobile robot egomotion is presented, which relies on tracking contours in real-time images acquired with a calibrated monocular video system. After fitting an active contour to an object in the image, 3D motion is derived from the affine deformations suffered by the contour in an image sequence. More than one object can be tracked at the same time yielding some different pose estimations. Then, improvements in pose determination are achieved by fusing all these different estimations. Inertial information is used to obtain better estimates, as it introduces in the tracking algorithm a measure of the real velocity. Inertial information is also used to eliminate some ambiguities arising from the use of a monocular image sequence. As the algorithms developed are intended to be used in real-time control systems, considerations on computation costs are taken into account.
\end{abstract}

\section{Introduction}

In feature-based techniques, points have been the traditional source of information for image-based tracking algorithms. More complex structures such as lines and corners have also been used. Recently, active contours fitted to image objects have been proposed as tracking features [1], especially in applications not requiring a high precision, as it is the case of robot navigation. Tracking active contours provides an estimate of robot position and orientation ("pose", for short), by relating it to the changes of the contour projection in the image plane. A calibrated monocular vision system is used and the image projection is modeled using the weak-perspective camera model. 
The algorithm for pose recovery has the following four steps. First, the active contour has to be initialized. One common way to represent active contours is by using b-splines [2]. In this work, initialization of the b-spline is manually performed by an operator. When corners are present, the use of a corner detector [3] improves the initial adjustment. Automatic initialization techniques have been proposed [4] and tested with good results. Since we are assuming weak perspective, only affine deformations of the initial contour will be allowed by the tracker and, therefore, the initialization process is important as it determines the family of affine deformations that the contour will be allowed to adjust to.

Second, a tracking algorithm recognizes the new position of the contour in the image and computes the change in position. A Kalman filter is used to integrate this new measurement with the history of previous ones. Since an affine deformation of a b-spline can be parameterized using a shape vector [5], this is used as the state vector for the filter. The shape vector is a set of 6 parameters representing the deformation of the b-spline independently of the complexity and the number of control points of the b-spline used. Tracking one contour provides an estimation of its shape vector $S$ and its associated covariance matrix $\Gamma_{S}$.

The tracking strategy is here enriched by using information provided by inertial sensors. Tracking at low velocities is known to be a favorable case, while tracking usually fails at high velocity rates. Using the good conditioning of inertial sensors precisely to detect relative high velocities, the tracking algorithm is improved by introducing the measured dynamics.

Third, from the shape vector $S$, the 3D camera pose is computed. It has been proved [6] that it is possible to obtain rotation and translation information from the shape vector by using SVD decomposition techniques. Using these techniques, the difference between the initial and the current pose is computed. It is worth noting that this is not an incremental method and, consequently, it does not suffer from the typical accumulative bias produced by signal integration in odometry and inertial-based navigation systems.

Finally, by fusing the information provided by the several contours available in the image, the 3D robot pose is obtained. Rotations are absolute values, whereas translations are recovered up to the scaled depth. Inertial measurements are useful also here to solve some ambiguities in the result arising from the use of a monocular vision system.

The paper is structured as follows. The next section explains how to obtain 3D information from tracked active contours. Section 3 gives the main ideas to understand the tracking of one contour and how inertial data is combined with contour tracking, and the next section makes some considerations to be taken into account in the redesign of the tracking algorithms to allow for the simultaneous tracking of several active contours. Section 5 describes how inertial information helps to solve some ambiguities of the recovered pose. The results of two experiments are presented in Section 6 and, finally, some conclusions and the envisaged future work are discussed in Section 7. 


\section{Obtaining 3D pose from the deformation of contours}

Under weak-perspective conditions, the rigid motion of a $3 \mathrm{D}$ contour $\mathbf{D}(s)$ relative to a reference contour $\mathbf{D}_{\mathbf{0}}(s)$,

$$
\mathbf{D}(s)=R \mathbf{D}_{\mathbf{0}}(s)+\mathbf{T},
$$

projects on the camera as an affine deformation of the projection of the reference contour, namely the template, that can be expressed as:

$$
\mathbf{d}(s)-\mathbf{d}_{\mathbf{0}}(s)=(M-I) \mathbf{d}_{\mathbf{0}}(s)
$$

where $\mathbf{I}$ is the identity matrix,

$$
\begin{aligned}
& \mathbf{M}=\frac{Z_{0}}{R_{33} Z_{0}+T_{Z}}\left[\begin{array}{ll}
R_{11} & R_{21} \\
R_{21} & R_{22}
\end{array}\right], \\
& \mathbf{t}=\frac{1}{R_{33} Z_{0}+T_{Z}}\left[\begin{array}{c}
Z_{0} R_{13}+T_{x} \\
Z_{0} R_{23}+T_{y}
\end{array}\right],
\end{aligned}
$$

$\mathbf{R}_{\mathbf{i j}}$ are the elements of the $3 \mathrm{D}$ rotation matrix $\mathbf{R}, \mathbf{T}_{\mathbf{i}}$ are the elements of the translation vector $\mathbf{T}$ and $Z_{0}$ is the distance from the template $\mathbf{D}_{\mathbf{0}}(s)$ to the camera.

Using the Euler notation to represent the rotation matrix,

$$
\mathbf{R}=\mathbf{R}_{\mathbf{z}}(\phi) \mathbf{R}_{\mathbf{x}}(\theta) \mathbf{R}_{\mathbf{z}}(\psi),
$$

equation (1) can be rewritten as

$$
\begin{aligned}
\mathbf{M}= & \left.\left.\left.\frac{Z_{0}}{T_{z}+R_{33} Z_{0}} \mathbf{R}_{\mathbf{z}}\right|_{\mathbf{2}}(\phi) \mathbf{R}_{\mathbf{x}}\right|_{\mathbf{2}}(\theta) \mathbf{R}_{\mathbf{z}}\right|_{\mathbf{2}}(\psi)= \\
= & \left.\left.\frac{Z_{0}}{T_{z}+R_{33} Z_{0}} \mathbf{R}_{\mathbf{z}}\right|_{\mathbf{2}}(\phi)\left[\begin{array}{cc}
1 & 0 \\
0 & \cos \theta
\end{array}\right] \mathbf{R}_{\mathbf{z}}\right|_{\mathbf{2}}(\psi)
\end{aligned}
$$

and,

$$
\mathbf{M M}^{T}=\left.\left.\mathbf{R}_{\mathbf{z}}\right|_{\mathbf{2}}(\phi)\left[\begin{array}{cc}
L & 0 \\
0 & L \cos ^{2} \theta
\end{array}\right] \mathbf{R}_{\mathbf{z}}\right|_{\mathbf{2}} ^{-1}(\phi)
$$

where

$$
\mathbf{L}=\left(\frac{Z_{0}}{T_{z}+R_{33} Z_{0}}\right)^{2}
$$

This last equation shows that $\theta$ can be computed from the ratio of eigenvalues of $\mathbf{M} \mathbf{M}^{\mathbf{T}}$, namely $\left(\lambda_{1}, \lambda_{2}\right)$,

$$
\cos \theta=\sqrt{\frac{\lambda_{2}}{\lambda_{1}}},
$$

where $\lambda_{1}$ is the largest eigenvalue and $\lambda_{2}$ the smallest one. The angle $\phi$ can be extracted from the eigenvectors of $\mathbf{M M}^{T}$. The eigenvector $\mathbf{v}_{\mathbf{1}}$ with largest eigenvalue equals the first column of $\left.\mathbf{R}_{\mathbf{z}}\right|_{\mathbf{2}}(\phi)$,

$$
\mathbf{v}_{\mathbf{1}}=\left[\begin{array}{c}
\cos \phi \\
\sin \phi
\end{array}\right] \text {. }
$$


Isolating $\left.\mathbf{R}_{\mathbf{z}}\right|_{\mathbf{2}}(\psi)$ from equation (4),

$$
\left.\mathbf{R}_{\mathbf{z}}\right|_{\mathbf{2}}(\psi)=\left.\left(R_{33}+\frac{T_{z}}{Z_{0}}\right)\left[\begin{array}{cc}
1 & 0 \\
0 & \frac{1}{\cos \theta}
\end{array}\right] \mathbf{R}_{\mathbf{z}}\right|_{\mathbf{2}}(-\phi) \mathbf{M},
$$

and observing, from equation (5), that

$$
R_{33}+\frac{T_{z}}{Z_{0}}=\frac{1}{\sqrt{\lambda_{1}}}
$$

we can find $\sin \psi$ and then $\psi$.

Once the angles $\psi, \theta, \phi$ are known, the rotation matrix $\mathbf{R}$ can be computed as in equation (3).

The scaled translation along $\mathrm{Z}$ can be computed as

$$
\frac{T_{z}}{Z_{0}}=\frac{1}{\sqrt{\lambda_{1}}}-R_{33} .
$$

The rest of the components of the 3D translation vector can be computed from $\mathbf{t}$ and $\mathbf{R}$ using equation (2),

$$
\begin{aligned}
& \frac{T_{x}}{Z_{0}}=\frac{t_{x}}{f \sqrt{\lambda_{1}}}-R_{13}, \\
& \frac{T_{y}}{Z_{0}}=\frac{t_{y}}{f \sqrt{\lambda_{1}}}-R_{23} .
\end{aligned}
$$

\section{$3 \quad$ Using inertial information in tracking}

The objective of tracking is to follow an object contour along a sequence of images. Due to its representation as a b-spline, the contour is divided naturally into sections, each one between two consecutive nodes. For the tracking, some interest points are defined along each contour section. Passing through each point and normal to the contour, a line segment is defined as shown in Figure 1. The search for edge elements (called "edgels") is performed only for the pixels under these normal segments, and the result is the Kalman measurement step. This allows the system to be quick, since only local image processing is carried out, avoiding the use of high-cost image segmentation algorithms.

Once edge elements along all search segments are located, the Kalman filter fuses this measured contour with that predicted from previous history, so that the resulting shape vector is always an affine deformation of the initial contour.

The length of the search segments is determined by the covariance estimated in the preceding frame by the Kalman filter. This is done by projecting the covariance matrix into the line normal to the contour at the given point. If tracking is finding good affine transformations that explain changes in the image, the covariance decreases and the search segments shrank. On the one hand, this is a good strategy as features are searched more locally and noise in image affects less the system. But, on the other hand, this solution is not the best for tracking large changes in image projection.

Large changes in image position can be produced by quick movements of the camera. As mentioned above, a weak-perspective model is used for camera modeling. To fit the model, the camera field-of-view has to be narrow. In such 
a situation, distant objects may produce important changes in the image also in the case of small movements of the camera.

For each search segment normal to the contour, the scale factor is computed as

$$
\mathbf{E}=\sqrt{\mathbf{N}^{T}\left(\mathbf{H P H}^{T}\right) \mathbf{N}}
$$

where $\mathrm{N}$ are the normal line coordinates, $\mathrm{H}$ is the measurement vector and $\mathrm{P}$ is the $6 \times 6$ top corner of the covariance matrix. Detailed information can be found in [5].

Note that, as covariance is changing at every frame, the search scale has to be recalculated also for each frame. It is also worth noting that this technique produces different search ranges depending on the orientation of the normal, taking into account the directional estimation of covariance of the Kalman filter.

In what follows, we explain how inertial information is used to adapt the search ranges locally on the contour by taking into account the measured dynamics. Consider a 3 d.o.f. inertial sensor providing coordinates $(\mathrm{x}, \mathrm{y}, \theta)$. To avoid having to perform a coordinate transformation between the sensor and the camera, the sensor is placed below the camera with their reference frames aligned. In this way, the $\mathrm{x}$ and $\mathrm{y}$ coordinates of the inertial sensor map to the $\mathrm{z}$ and $\mathrm{x}$ camera coordinates, respectively, and rotations take place about the same axis. Sensed movement can be expressed then as a translation

$$
\mathbf{T}=\left[\begin{array}{c}
v_{x} \\
0 \\
v_{z}
\end{array}\right]
$$

and a rotation

$$
\mathbf{R}=\left[\begin{array}{ccc}
\cos \omega_{y} & 0 & \sin \omega_{y} \\
0 & 1 & 0 \\
-\sin \omega_{y} & 0 & \cos \omega_{y}
\end{array}\right]
$$

Using equations $(1,2)$ with equations $(11,12)$ sensed data can be expressed in shape vector space as

$$
\begin{gathered}
M_{11}=\frac{Z_{0}}{Z_{0} R_{33}+T_{z}} R_{11}=\frac{Z_{0}}{Z_{0} \cos \omega_{y}+v_{z}} \cos \omega_{y} \\
M_{21}=\frac{Z_{0}}{Z_{0} R_{33}+T_{z}} R_{21}=0 \\
M_{12}=\frac{Z_{0}}{Z_{0} R_{33}+T_{z}} R_{12}=0 \\
M_{22}=\frac{Z_{0}}{Z_{0} R_{33}+T_{z}} R_{22}=\frac{Z_{0}}{Z_{0} \cos \omega_{y}+v_{z}} \\
t_{1}=\frac{1}{Z_{0} R_{33}+T_{z}}\left(Z_{0} R_{13}+T_{x}\right)=\frac{1}{Z_{0} \cos \omega_{y}+v_{z}}\left(-Z_{0} \sin \omega_{y}+v_{x}\right) \\
t_{2}=\frac{1}{R_{33} Z_{0}+T_{z}}\left(Z_{0} R_{23}+T_{y}\right)=0
\end{gathered}
$$

As the objective is to scale covariance, denominators can be eliminated in equations $(13-15)$. These equations can be rewritten in shape vector form as 


$$
\mathbf{S}=\quad \begin{array}{rrrrrr}
\left(\begin{array}{cccccc}
t_{1} & 0 & M_{11}-1 & M_{22}-1 & 0 & 0
\end{array}\right)= \\
\left(\begin{array}{llllll}
-Z_{0} \sin \omega_{y}+v_{x} & 0 & -v_{z} & Z_{0}\left(1-\cos \omega_{y}\right)-v_{z} & 0 & 0
\end{array}\right)
\end{array}
$$

For small rotational velocities, $\sin \omega_{y}$ can be approximated by $\omega_{y}$ and, thus,

$$
\mathbf{S}=\left(\begin{array}{llllll}
-Z_{0} \omega_{y}+v_{x} & 0 & -v_{z} & Z_{0} \omega_{y}^{2} / 2-v_{z} & 0 & 0
\end{array}\right)
$$

The inertial sensor gives the $\mathrm{x}$ direction data in the range $\left[v_{x \min } . . v_{x \max }\right]$. To simplify the notation, let us consider a symmetric sensor, i.e., $v_{x \min }=v_{x \max }$. Sensor readings can be rescaled to provide values in the range $\left[\bar{v}_{x \min } . . \bar{v}_{x \max }\right]$. A value $v_{x}$ provided by the inertial sensor can be rescaled using

$$
\overline{v_{x}}=\left|v_{x}\right| \frac{\bar{v}_{x \max }-\bar{v}_{x \min }}{v_{x \max }}+\bar{v}_{x \min }
$$

Through the same reasoning, shape vector parameters can be rescaled. For the first component we have

$$
t_{1 \max }=Z_{0} \omega_{y \max }+v_{x \max }
$$

and the expression

$$
\overline{t_{1}}=\left|t_{1}\right| \frac{\bar{t}_{1 \max }-\bar{t}_{1 \min }}{t_{1 \max }}+\bar{t}_{1 \min }=\left|t_{1}\right| f_{t_{1}}+\bar{t}_{1 \min }
$$

Inertial information can be added now by scaling the current covariance sub-matrix by a matrix representing the scaled inertial data as follows

$$
\mathbf{E}=\sqrt{\mathbf{N}^{T}\left(\mathbf{H V P V}^{T} \mathbf{H}^{T}\right) \mathbf{N}}
$$

where $\mathbf{V}$ is the scaled measurement matrix for the inertial sensing system defined as

$$
\mathbf{V}=\left[\begin{array}{cccccc}
t_{1} & & . . & & & 0 \\
& t_{2} & & & & \\
\cdot & M_{11}-1 & & & \cdot \\
\cdot & & & M_{22}-1 & & \cdot \\
0 & . . & & M_{12} & \\
0 & & & & M_{21}
\end{array}\right]\left[\begin{array}{c}
f_{t_{1}} \\
f_{t_{2}} \\
f_{M_{11}} \\
f_{M_{22}} \\
f_{M_{12}} \\
f_{M_{21}}
\end{array}\right]+\left[\begin{array}{c}
\bar{t}_{1 \min } \\
\bar{t}_{2 \text { min }} \\
\bar{M}_{11 \text { min }} \\
\bar{M}_{22 \text { min }} \\
\bar{M}_{12 \text { min }} \\
\bar{M}_{21 \text { min }}
\end{array}\right]
$$

For testing purposes, the minimum and maximum values of all components have been set to 1 and 2 , respectively.

\section{From one to $\mathrm{n}$ trackers}

Tracking of one contour provides, in principle, enough information to obtain an estimate of the change in robot pose from the initial frame. Although the tracking algorithm is designed to be robust to some kinds of partial occlusions, 
there are some situations where the tracker gets necessarily lost and fails to recover the true 3D pose. For example, under an automatic initialization scheme, a contour may be fitted to a moving object. In this case, the estimate would not be the robot movement, but the relative motion between the moving object and the vehicle (note that, if the camera is known not to be moving, then the object movement is recovered). An active contour can also be initialized to fit a very close object, or a robot trajectory may take objects closer to the camera. In such situations, perspective effects appear. Affine transformations are then not enough to encode the deformations of the contours in the image and errors in the recovered pose become larger. Moreover, due to the camera model adopted, the difference in depth of the points that form the contour must be small, as the contour is taken to be a planar one. Effects of apparent contours [7] in the image may produce large errors in estimates.

By tracking simultaneously more than one contour in the image, several pose estimates are obtained. It is expected that, by fusing all these estimates, improvements in both pose estimation and tracking robustness will be obtained.

Since all contours deform as a result of the same camera motion, one could easily think of sharing the same Kalman filter state between all contours. However, the shape vector of each contour depends on the distance from the contour to the camera, which may be different for each contour. Moreover, taking into account the differences in initialization, as well as the disruptive phenomena explained above, it turns out that this is not a good option. Trackers should be as independent as possible to provide separate estimates, most of which will hopefully be free of the disruptions in image projection mentioned above. In this way, outliers could be spotted and a reliable estimate could be derived by fusing the estimates from the remaining trackers.

Following this idea, the software design is shown in Figure 2.

\section{$5 \quad$ Estimating 3D pose}

From each tracker a shape vector and its covariance is obtained. The system pose estimation is computed using all the individual estimations.

Section 2 has defined a method to translate tracked data from shape vector space into motion parameters in $3 \mathrm{D}$ space. One option to combine all these informations is to use statistical methods. Statistical fusion [8] is proposed as a first approach.

Assuming no outliers among the tracked contours, all the estimates can be combined to obtain a better estimation of the pose. We propose to use the trace of the covariance matrix as an approximation of the variance of each pose. An individual estimation of pose is obtained from each contour at frame rate.

For simplicity we analyze the case of two estimations. For each degree of freedom $\left(T_{x} T_{y}, T_{z}, \phi, \theta, \psi\right)$, the new estimated value, for example $T_{x}$, is based on estimations $T 1_{x}$ and $T 2_{x}$ from two different trackers, and can be computed using:

$$
\begin{gathered}
T_{x}=\left[\frac{\sigma_{2}^{2}}{\sigma_{1}^{2}+\sigma_{2}^{2}}\right] T 1_{x}+\left[\frac{\sigma_{1}^{2}}{\sigma_{1}^{2}+\sigma_{2}^{2}}\right] T 2_{x} \\
\frac{1}{\sigma^{2}}=\frac{1}{\sigma_{1}^{2}}+\frac{1}{\sigma_{2}^{2}}
\end{gathered}
$$


It is easy to prove that not all translations and rotations will be sensed with the same resolution. Translations parallel to the image plane are better sensed as they produce greater changes in image than translations normal to the image plane. The same reason explains that rotations about an axis perpendicular to the image plane are better sensed than the ones about axes parallel to the image plane. This can be seen in Figure 3, where a static camera tracks a moving contour. In this experiment a synthetic image is used in order to avoid measuring noise from the acquisition system. As predicted, in translation (Figure 3(a))

depth is worst estimated, yielding greater variance. In rotational recovered parameters (Figure 3(b)), Z varies less than $\mathrm{X}$ and $\mathrm{Y}$.

Using monocular vision one can observe that different real 3D movements result in the same, or very similar, projections on the image plane. This is due to both finite resolution and the well-known projection ambiguities.

One of these ambiguities is the so called Necker reversal ambiguity. It is explained in Figure 4. Take a point rotating a certain angle about a point in a plane parallel to the image plane. Its projection in the image will be very similar to that of another point, in mirror position from the parallel plane, rotating the same angle but in the opposite direction.

When projection effects are big, the sign of the angle is easy to recover. When these effects diminish, as it is the case of this work, the direction becomes undetermined. This can be determined using an inertial sensor, as it provides the sign of the movement.

Other ambiguities should be solved, or at least limited using inertial data. However, it requires more precise and robust data from the inertial sensors, which we have not available at the moment.

\section{Experiments and results}

\subsection{Pose estimation with 2 tracked contours}

To evaluate the performance of the tracker information fusion, synthetic images are used. In this way, errors produced by the tracking system can be easily isolated and the fusion performance can be better evaluated.

A sequence of 100 frames is used simulating a camera rotating about the $\mathrm{Z}$ axis. Each successive frame is rotated by 1 degree, thus the total rotation is 100 degrees.

Different initializations are used for each contour. As can be seen in Figure 5, the number of control points used to define the right contour is twice the number used for the left one.

Recovered rotations for both contours are shown in Figure 6. As rotation in ZXZ Euler representation is not intuitive, angles are expressed in XYZ form. Rotation about the $\mathrm{Z}$ axis is quite well recovered through the sequence of frames. In the case of rotations about the $\mathrm{X}$ and $\mathrm{Y}$ axes, just noise is recovered as the camera does not move along these directions.

Effects of different initializations can be seen in Table 1. As more points are used to model the right contour, the standard deviation decreases and becomes lower than in the left contour.

As tracking performance improves, the computed covariance also improves, providing a lower mean value of covariance and a lower standard deviation. This 
can be seen in Table 2, meaning that tracking with more points is more stable.

Using statistical fusion, both estimates can be combined to obtain a better estimate. Results of fusion are shown in Figure 7. As can be seen, values for rotation follow real motion. Noise from single estimations is more stable, and covariance is also stable as the figure is taken at a very high zooming rate. This is important, because it shows that covariance can be used as a quality factor for single pose recovery from the tracking algorithm.

\subsection{Improved tracking with inertial information}

For this work we use a RobuCab Mobile Robot [9]. It can be used in two modes: car-like navigation and bi-directional driving. As can be seen in Figure 8, it is a relatively big mobile vehicle with capacity for up to four people.

For simplicity of the control system, the car-like driving option is used, but better results should be obtained under bi-direction driving mode as the maximum turning angle would increase. In this vehicle we mount a monocular vision system with the described 6 d.o.f. tracking system. A Gyrostar inertial sensor, from Murata, is used to measure rotations about the $\mathrm{Y}$ axis. To measure $\mathrm{X}$ and $\mathrm{Z}$ linear accelerations, an ADXL dual accelerometer from Analog Devices is used. All these sensors are connected to a dedicated board with an AVR processor used to make A/D conversions, PWM decoding and time integration. It has also a thermometer for thermal data correction. This 'intelligent' sensor provides not only changes in velocity, but also mean velocity and position. Drift, typical in this kind of computations, is reset periodically with the information obtained by fusion of the other sensors. This board shares memory with a MPC555 board, which is connected through a CAN bus to the control and vision processing PC. All the system runs under a real-time Linux kernel in a Pentium $233 \mathrm{MHz}$ industrial box. A novel approach to distributed programming [10] has been used to program robot control as well as for the intercommunication of control and vision processes, taking advantage of the real time operating system. Figure 9 shows the hardware components and their interconnections.

The shape vector we use models all plane deformations resulting from 3D motions. It could be changed in this experiment in order to model only 3 d.o.f. movements [6]. However, as the robot real movements are in 6 parameter space, mainly due to floor rugosity and vehicle dampers, the whole shape vector is used.

In this experiment the robot is in autonomous driving mode, following a filoguided path. In this way, the trajectory can be easily repeated, thus allowing us to perform several experiments with very similar conditions. The path followed consists of a straight line segment, a curve and another straight line.

To use inertial data within the visual tracker, we need to compute the vector $\mathrm{S}$ in equation (16). For this, it is necessary to have an estimation of $Z_{0}$, i.e., the initial distance of the target to the sensor set. In this experiment, this value has been set by the operator, but in future work we expect to derive it automatically by relating the difference in distance measured by the inertial sensor to the scaled target depth obtained by the vision process.

First, the algorithm without inertial information is used. On the first straight segment, the contour is well followed, but as can be seen in Figure 10(a), when turning takes place and the contour moves quicker in the image plane, it loses the real object and the covariance increases. 
Second, the algorithm adding inertial information to the tracker is used. In this experiment, tracking does not lose the target and finishes the sequence giving good recovered pose values. As can be seen in the covariance representation in Figure 10(b), covariance increases at the beginning of the turning, but decreases quickly, showing that tracking has fixed the target despite its quick translation across the image.

As can be easily seen, if we use more images trying to track the target while moving towards it, the contour leaves the image range. Another contour should be initialized in order to be able to continue egomotion recovery in such situation.

\section{Conclusions}

This article presents a novel approach to enrich visual tracking of active contours with information provided by inertial sensors. First, the tracking strategy has been modified to include inertial data. In this way, contour search in the new frame by taking into account its position in the preceding frame is made more robust, thus allowing tracking at higher velocities of the video data.

The paper also presents a framework to extend the method to be able to use multiple contours and shows the way to estimate the 3D pose from several tracked contours. A preliminary approach to fuse several estimations has been presented. Taking advantage of the inertial sensing of the rotation sign, the Necker reversal ambiguity has been solved.

Initial experiments have been performed to prove correctness in some reduced situations. Further work should include the change of the fusion strategy to take into account directional information in the covariance matrix.

Improvements in the quality of the data obtained from inertial sensors will allow to reduce, or eliminate, some other ambiguities in the pose recovery algorithm. Moreover, by relating distance differences measured by the inertial sensor to scaled depths obtained by the vision system, a reasonable approximation of the $Z_{0}$ parameter should be provided automatically. Experiments using whole information provided by the inertial sensor set should be also performed. As pose recovery is up to 6 dof, experiments with complete 3D movements will be useful to test the feasibility of ego-motion recovery algorithms. To easily show algorithm performance next experiments should include ground truth.

\section{Acknowledgments}

Guillem Alenyà was supported by the Marie Curie FP5 program. Elisa Martínez and Carme Torras acknowledge support from the Spanish Science and Technology Council, project DPI2000-1352-C02-01.

\section{References}

[1] E. Martínez and C. Torras, Qualitative vision for the guidance of legged robots in unstructured environments, Pattern Recognition, 34(2001)15851599. 
[2] A. Blake, M. Isard, D. Reynard, Learning to track the visual motion of contours, Artificial Intelligence, 78(1995)101-134.

[3] C. Harris, M.A. Stephens, Combined corner and edge detector, Proceedings of 4th Alvey Vision Conference,1988, pp 147-151.

[4] T.Cham and R.Cipolla, Automated B-Spline curve representation incorporating $\mathrm{MDL}$ and error-minimizing control point insertion strategies, IEEE Trans. on Pattern Analysis and Machine Intelligence, 21(1999).

[5] A. Blake and M. Isard, Active contours, Springer, 1998.

[6] E. Martínez, Recovery of 3D structure and motion from the deformation of an active contour in a sequence of monocular images, $\mathrm{PhD}$ Thesis, 2000.

[7] R. Cipolla, The visual motion of curves and surfaces, Phil. Trans. R. Soc. Lond., 1998, 1103-1121.

[8] P. Maybeck, Stochastic Models, Estimation and Control, vol. 1, Academic Press Inc, 1979.

[9] http://www.robosoft.fr

[10] P. Pomiers, Integration policy for critical multilayered distributed robotics applications, IEEE Intelligent Vehicle Symposium, (2002). 


\section{List of Figures}

1 An image with a contour and its search segments highlighted. . . 13

2 Software design allowing the tracking of several contours. . . . . 14

3 3D pose evaluated in a free-of-motion contour. . . . . . . . . . 15

4 Necker reversal ambiguity appears when the perspective effects diminish. There is no ambiguity in the upper figure. But there is no difference between the image obtained when $A$ rotates to $A^{\prime}$ and the image recorded when $B$ rotates to $B^{\prime}$ in the lower figure. 16

5 Different initializations used in the experiment. . . . . . . . . 17

6 Recovered rotation. . . . . . . . . . . . . . . . . 18

$7 \quad$ Fusion result. . . . . . . . . . . . . . . . . . . . . . . 19

8 Mobile robot used in the experiment. . . . . . . . . . . . 20

9 Interconnection of system sensors and processing units. . . . . . . 21

10 Covariances resulting from tracking without using inertial information (a) and using it (b) . . . . . . . . . . . 22

\section{List of Tables}

1 Statistics for recovered XYZ rotation. . . . . . . . . . . 23

2 Statistics for covariance . . . . . . . . . . . . . . 24 


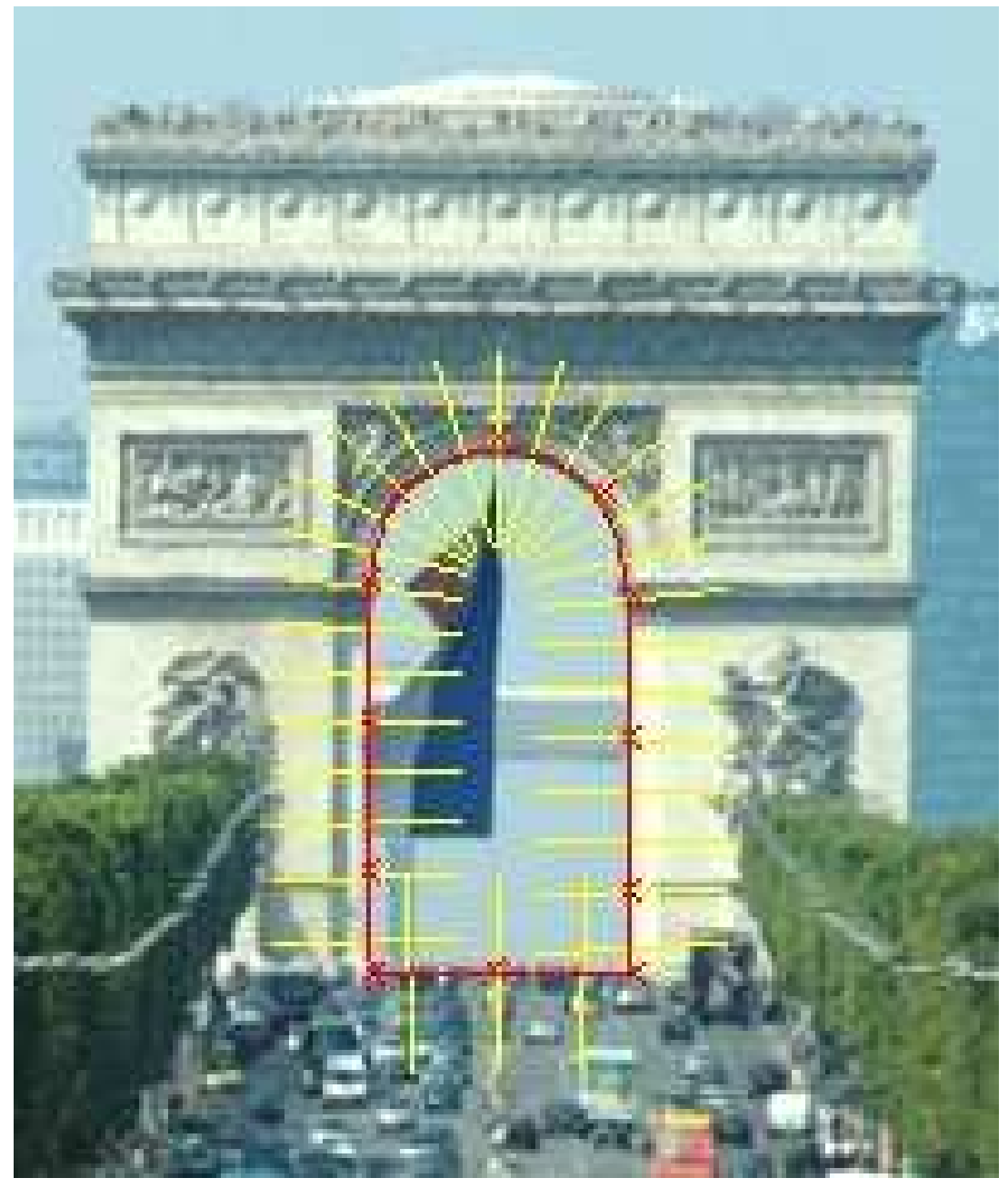

Figure 1: An image with a contour and its search segments highlighted. 


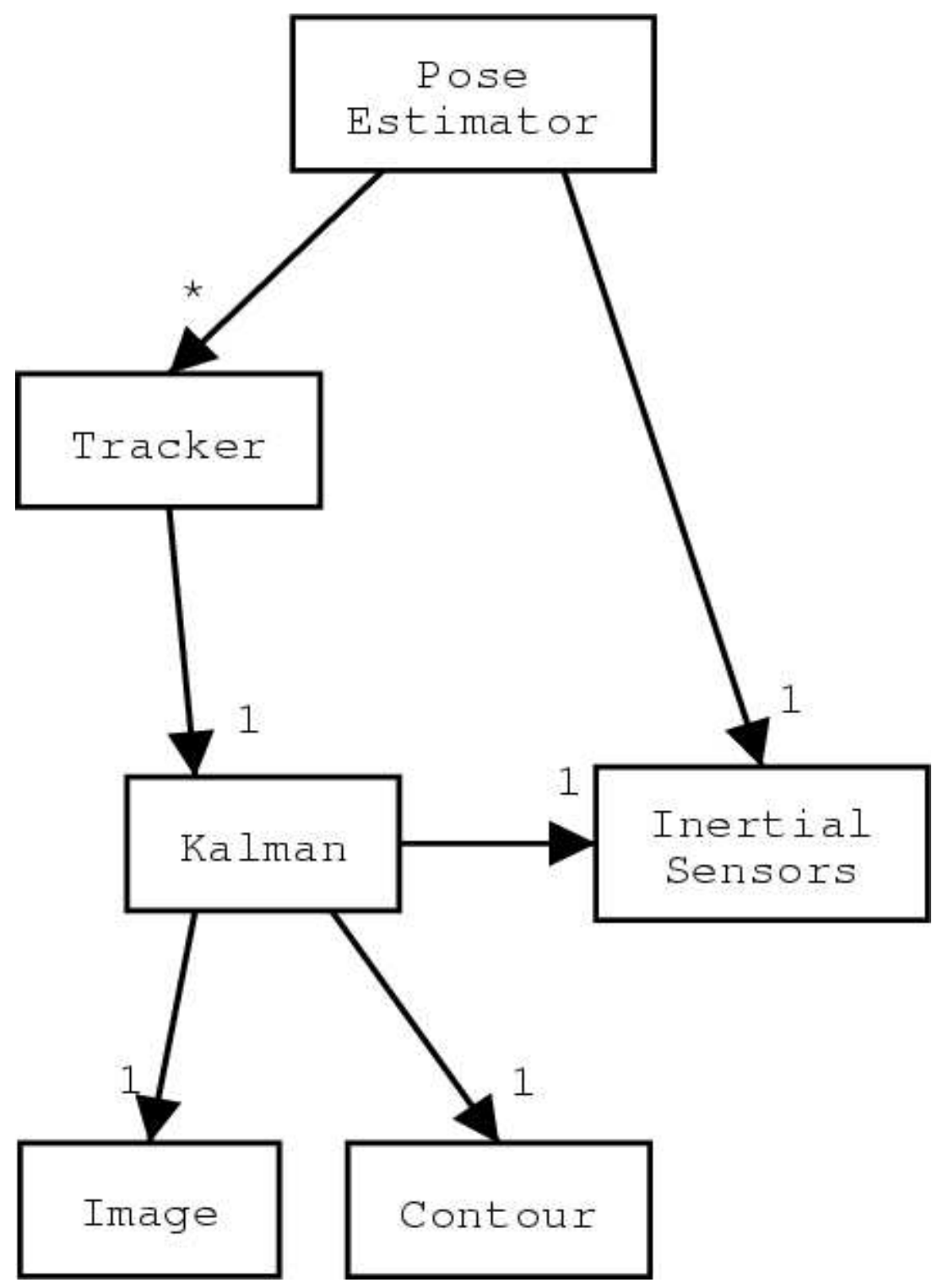

Figure 2: Software design allowing the tracking of several contours. 


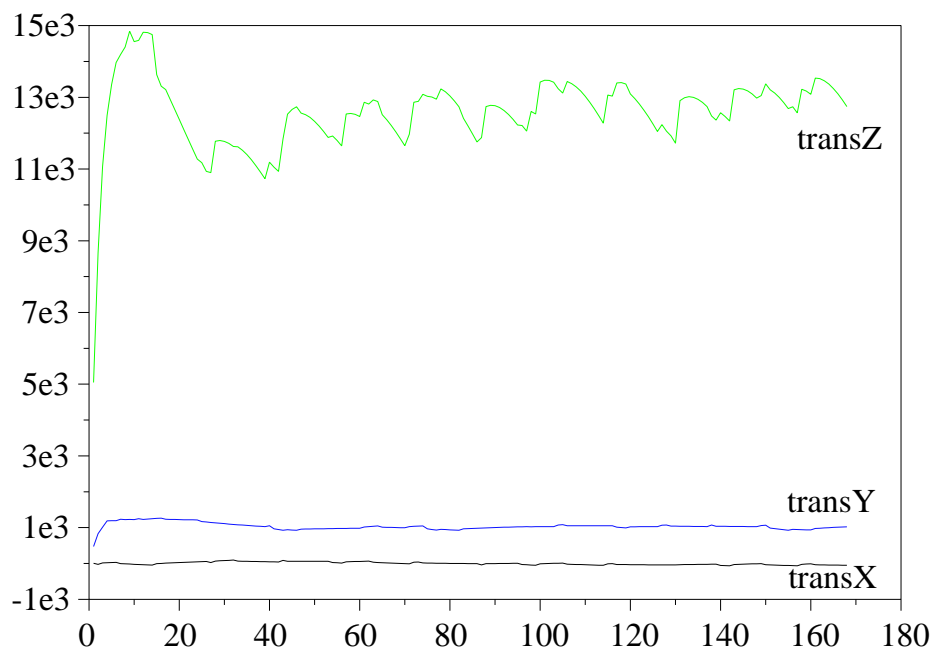

(a) Translation

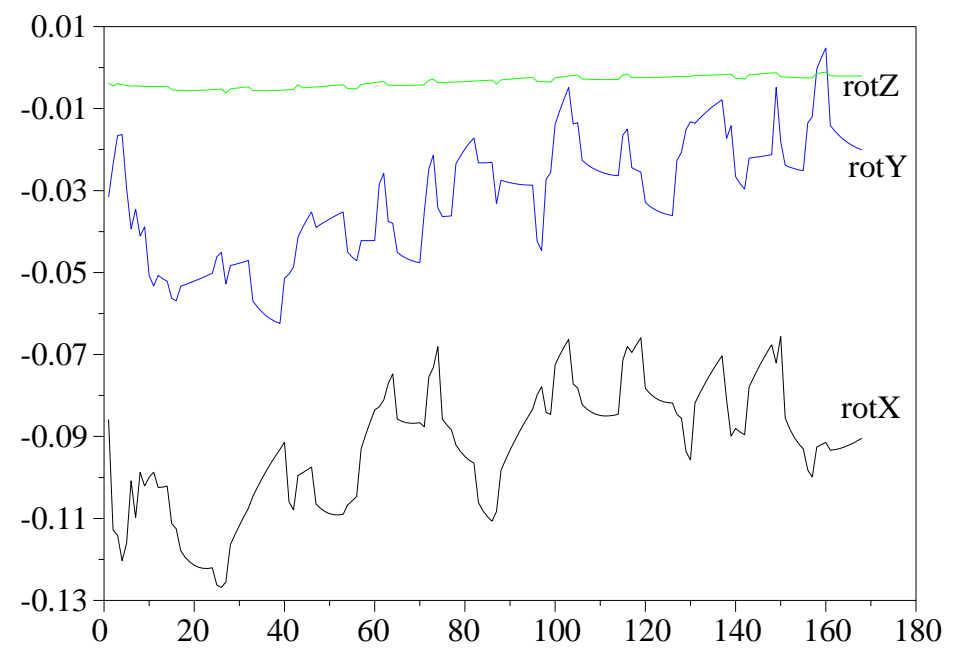

(b) Rotation

Figure 3: 3D pose evaluated in a free-of-motion contour. 


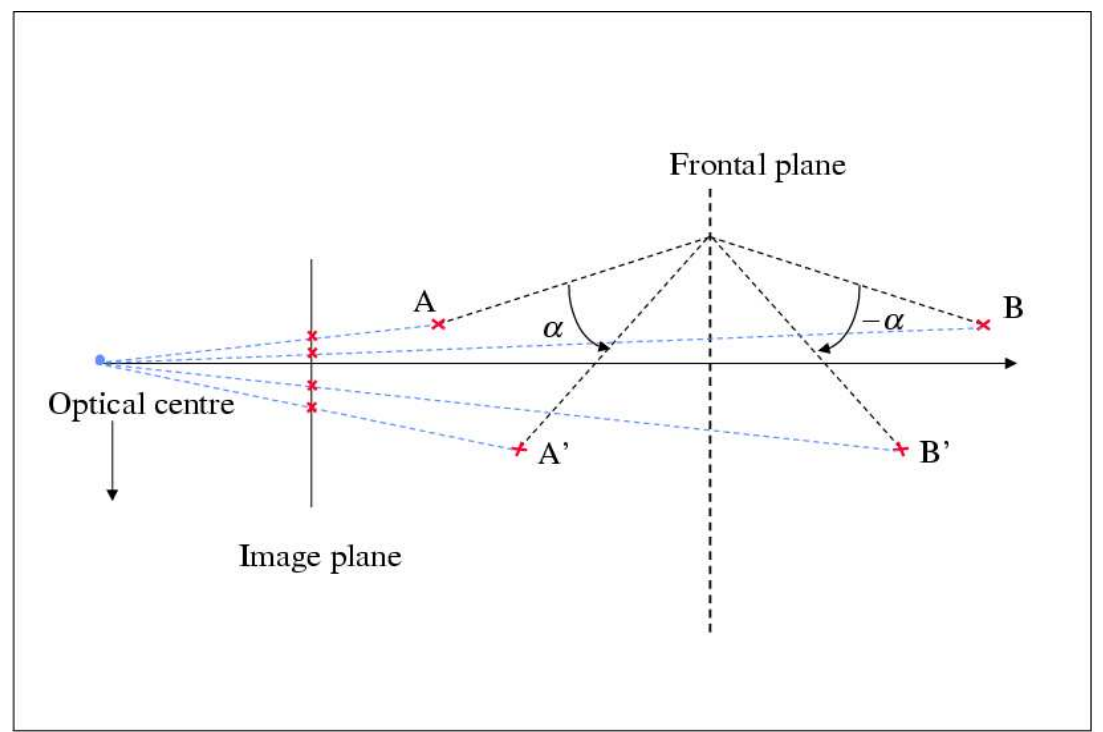

A.

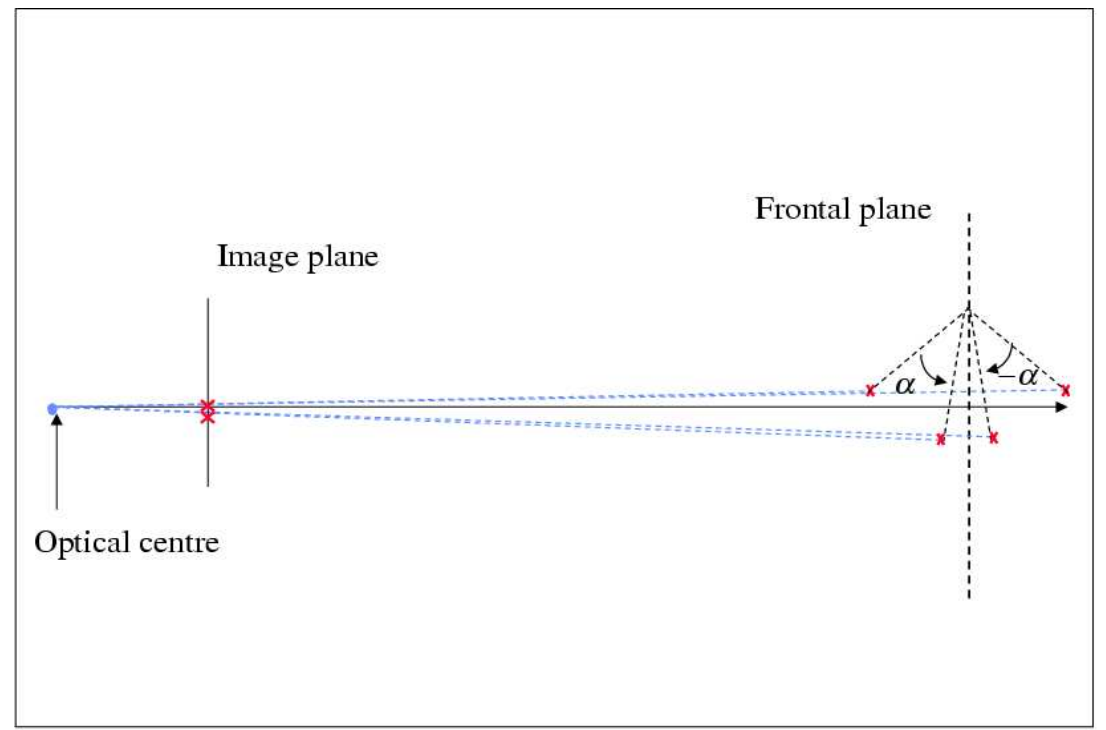

B.

Figure 4: Necker reversal ambiguity appears when the perspective effects diminish. There is no ambiguity in the upper figure. But there is no difference between the image obtained when $A$ rotates to $A^{\prime}$ and the image recorded when $B$ rotates to $B^{\prime}$ in the lower figure. 


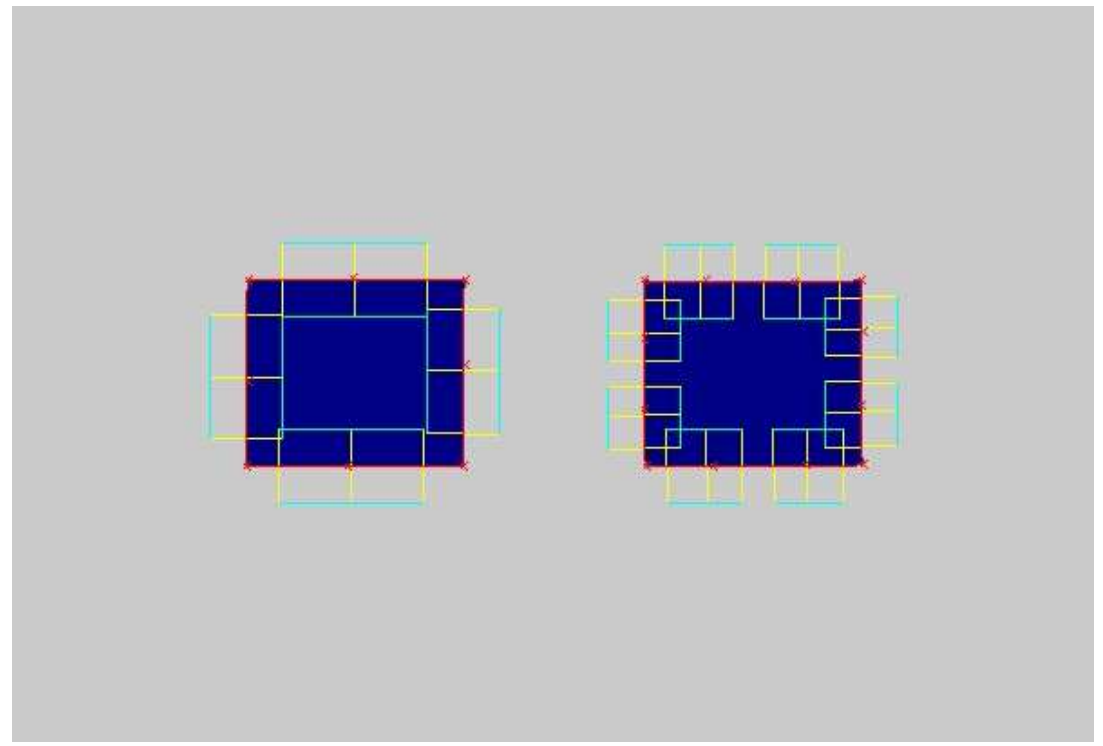

Figure 5: Different initializations used in the experiment. 


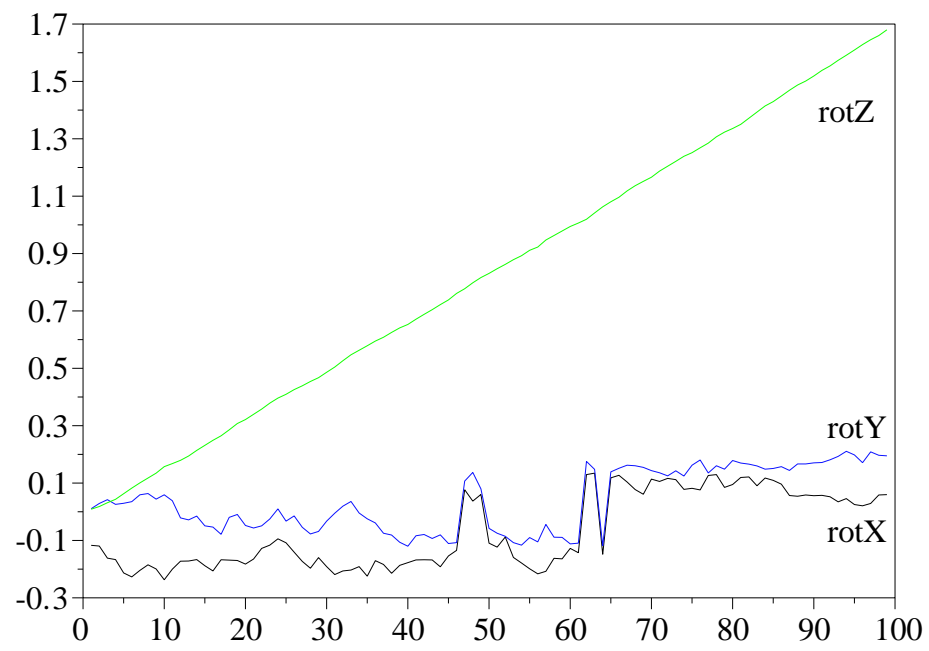

(a) Left

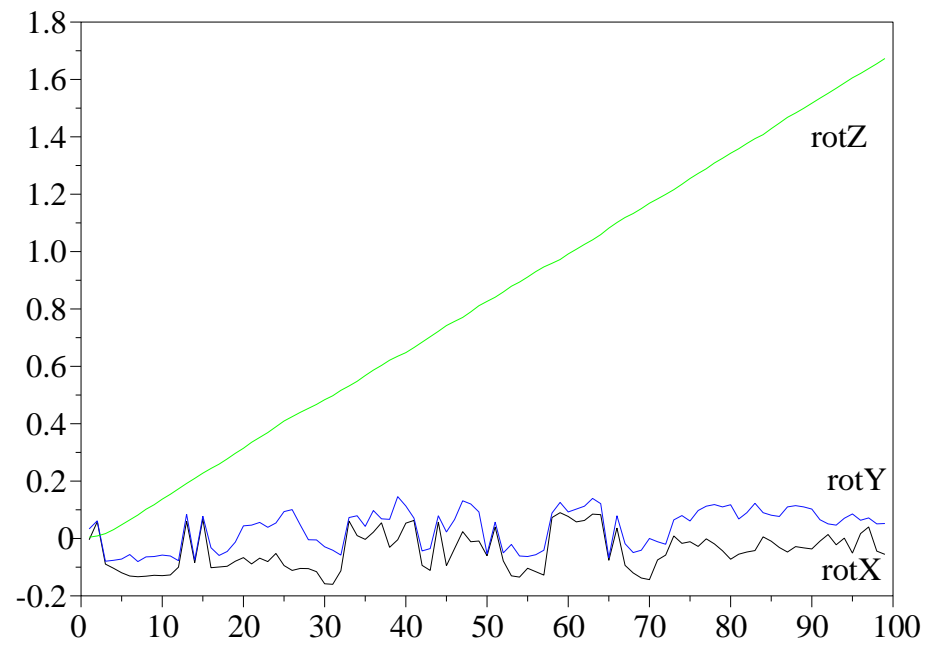

(b) Right

Figure 6: Recovered rotation. 


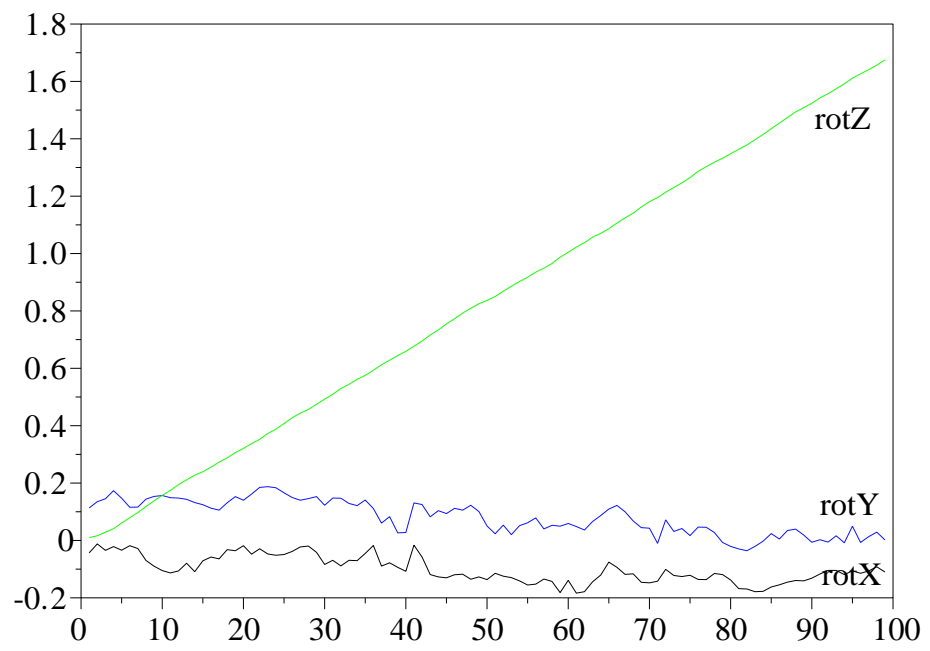

(a) Rotations

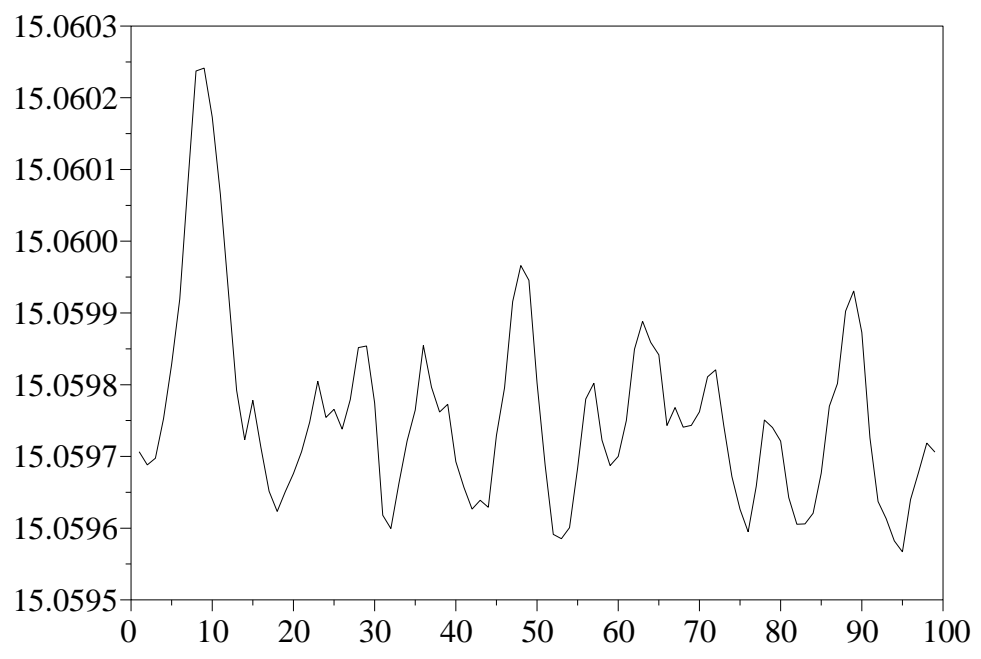

(b) Covariance

Figure 7: Fusion result. 


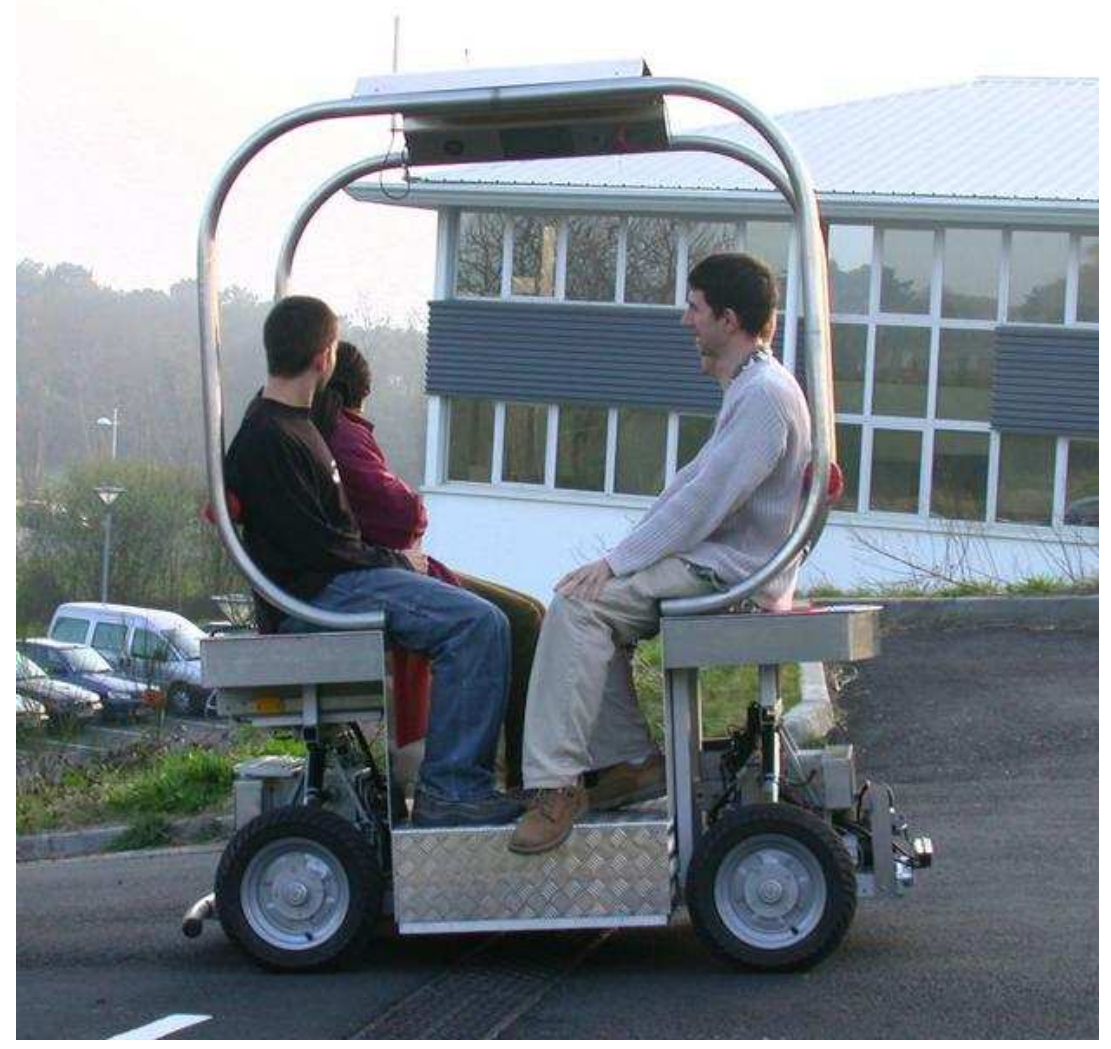

Figure 8: Mobile robot used in the experiment. 


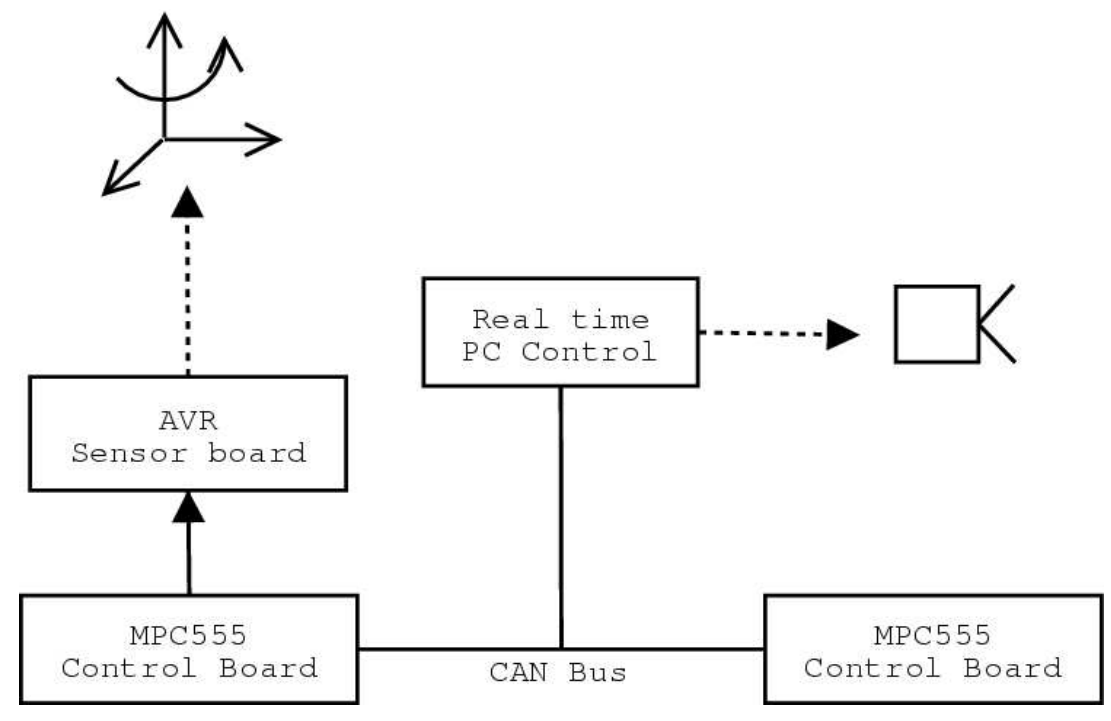

Figure 9: Interconnection of system sensors and processing units. 


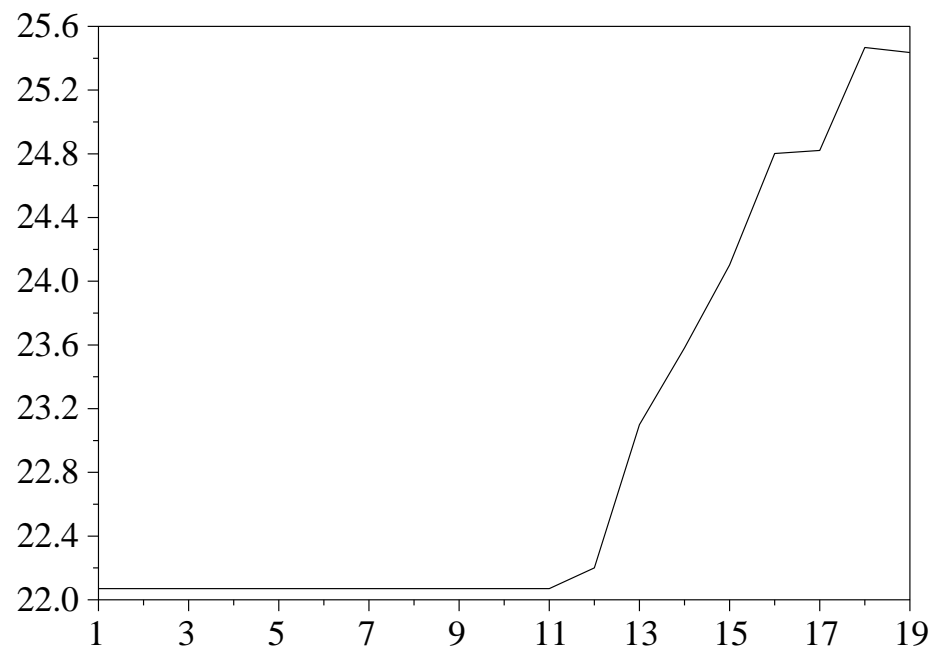

(a)

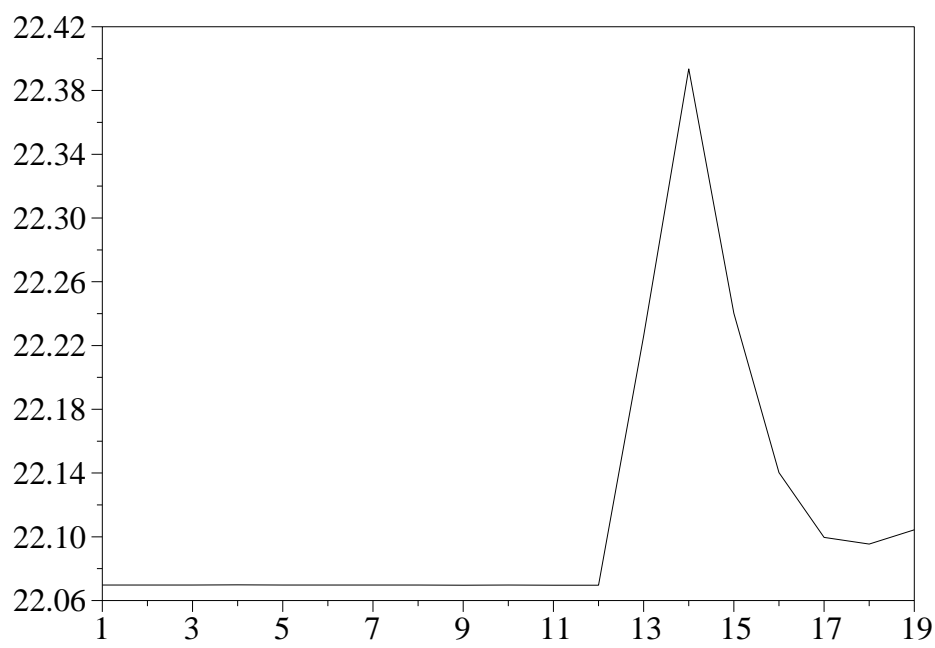

(b)

Figure 10: Covariances resulting from tracking without using inertial information (a) and using it (b). 


$$
\begin{array}{lcc}
\text { Left } & R x & R y \\
\text { Mean: } & -0.0684203 & 0.0397576 \\
\text { Std-dev }: & 0.1296382 & 0.1086573 \\
\text { Right } & & \\
\text { Mean : } & -0.0434605 & 0.0356667 \\
\text { Std-dev }: & 0.0676008 & 0.0679300
\end{array}
$$

Table 1: Statistics for recovered XYZ rotation. 


$\begin{array}{lcc} & \text { Left } & \text { Right } \\ \text { Mean : } & 20.917314 & 10.782572 \\ \text { Std-dev : } & 0.0001930 & 0.0000838\end{array}$

Table 2: Statistics for covariance 